\title{
Biophysical investigation of the interplay between the conformational species of domain-swapped GB1 amyloid mutant through real - time monitoring of amyloid fibrillation
}

Renuka Ranjan ${ }^{1,2}$, Nidhi Tiwari ${ }^{1,3}$, Arvind M. Kayastha ${ }^{2 *}$, Neeraj Sinha ${ }^{1 *}$

\author{
${ }^{1}$ Centre of Biomedical Research, SGPGIMS Campus, Raebareily Road, Lucknow-226014 \\ ${ }^{2}$ School of Biotechnology, Institute of Science, Banaras Hindu University, Varanasi-221005 \\ ${ }^{3}$ Department of Chemistry, Institute of Science, Banaras Hindu University, Varanasi-221005
}

* Authors to whom correspondence should be addressed;

(AMK) kayasthabhu@gmail.com

(NS)neeraj.sinha@cbmr.res.in 
Tris-Tricine SDS-PAGE to track low-molecular weight species during the process of aggregation.

An $18 \%$ tris-tricine SDS-PAGE gel image is shown in figure S1. Lane (M) shows marker protein ladder. Lane (1), (2), (3) and (4) shows the contents of the supernatant in GB1 HS\#124 ${ }^{\mathrm{F} 26 \mathrm{~A}}$ during the process of aggregation at time point $0 \mathrm{~h}, 12 \mathrm{~h}, 36 \mathrm{~h}$ and $84 \mathrm{~h}$ respectively.

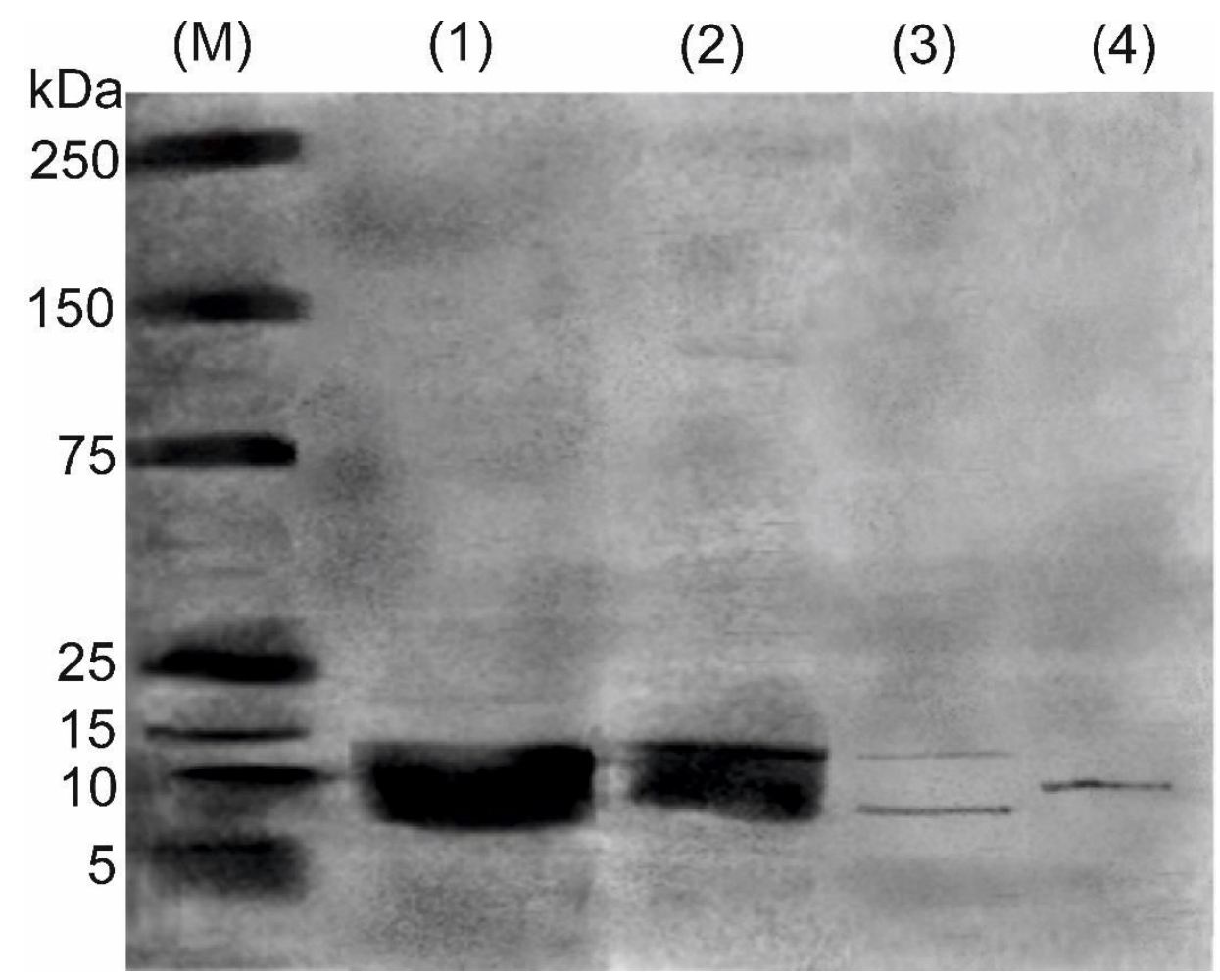

Figure S1: 18\% Tris-Tricine-SDS gel showing supernatant of GB1 HS\#124 ${ }^{\mathrm{F} 6 \mathrm{~A}}$ incubated under fibrillation conditions in respective lanes at time point (1) $0 \mathrm{~h} \mathrm{(2)} 12 \mathrm{~h} \mathrm{(3)} 36 \mathrm{~h} \mathrm{(4)} 84 \mathrm{~h}$. 
Side chain assignment of GB1HS\#124 F26A peptide was carried out using 1D ${ }^{1} \mathrm{H}$ NMR spectra and 2D ${ }^{1} \mathrm{H}-$ ${ }^{13} \mathrm{C}$ HSQC NMR spectra. These spectra were obtained at $25^{\circ} \mathrm{C}$ and $58^{\circ} \mathrm{C}$ and were compared to BMRB 5875 data set in order to assign chemical shifts to their respective ${ }^{1} \mathrm{H}$ and ${ }^{13} \mathrm{C}$ side chain atoms.

Table S1: Assignment of ${ }^{1} \mathrm{H}$ and ${ }^{13} \mathrm{C}$ chemical shift of GB1HS\#124 ${ }^{\mathrm{F26A}}$ peptide side chain at $58^{\circ} \mathrm{C}$ and $\mathrm{pH}=5.5$ (fibrillation condition) and changes in intensity of dimeric peaks by ${ }^{1} \mathrm{H}-{ }^{13} \mathrm{C}$ HSQC over the course of fibrillation.

\begin{tabular}{|c|c|c|c|c|c|}
\hline $\begin{array}{l}{ }^{1} \mathrm{H} \text { Chemical } \\
\text { Shift (in ppm) }\end{array}$ & $\begin{array}{l}{ }^{13} \mathrm{C} \text { Chemical } \\
\text { Shift (in ppm) }\end{array}$ & $\begin{array}{l}\text { Amino acid } \\
\text { residue }\end{array}$ & Assigned proton & $\begin{array}{l}\text { Assigned } \\
\text { Carbon }\end{array}$ & $\begin{array}{l}\text { Intensity } \\
\text { changes over } \\
\text { the course of } \\
\text { fibrillation }\end{array}$ \\
\hline-0.303 & 16.21 & V54 & HG21,HG22,HG23 & CG2 & Decrease \\
\hline 0.34 & 24.31 & $\mathrm{~L} 12$ & HD11,HD12,HD13 & $\mathrm{CD} 1$ & Decrease \\
\hline 0.37 & 22.67 & L12 & HD21,HD22,HD3 & $\mathrm{CD} 2$ & Decrease \\
\hline 0.608 & 19.01 & V5 & HG21,HG22,HG23 & CG2 & Decrease \\
\hline 0.616 & 20.65 & V30 & HG21,HG22,HG23 & CG2 & Decrease \\
\hline 0.72 & 18.97 & V54 & HG11,HG12,HG13 & CG1 & Decrease \\
\hline 0.94 & 22.3 & L7 & HD21,HD22,HD3 & $\mathrm{CD} 2$ & Decrease \\
\hline 0.96 & 23.1 & $\mathrm{~L} 7$ & HD11,HD12,HD13 & CD1 & Decrease \\
\hline 1.018 & 10.55 & I6 & HD11,HD12,HD13 & CD1 & Increase \\
\hline 1.03 & 18.51 & V5 & HG11,HG12,HG13 & CG1 & Decrease \\
\hline 1.041 & 21.41 & $\mathrm{~V} 30$ & HG11,HG12,HG13 & CG1 & Increase \\
\hline 1.054 & 15.01 & A23 & HB1,HB2,HB3 & $\mathrm{CB}$ & Increase \\
\hline 1.094 & 22.67 & V29 & HG11,HG12,HG13 & CG1 & Increase \\
\hline 1.11 & 18.54 & T16 & HG21,HG22,HG23 & CG2 & Increase \\
\hline 1.13 & 19.84 & V39 & HG11,HG12,HG13 & CG1 & Decrease \\
\hline 1.139 & 16.01 & A26 & HB1,HB2,HB3 & $\mathrm{CB}$ & Decrease \\
\hline 1.16 & 15.08 & I6 & HG21,HG22,HG23 & CG2 & Decrease \\
\hline 1.201 & 16.83 & V21 & HG21,HG22,HG23 & CG2 & Decrease \\
\hline 1.21 & 17.91 & V39 & HG21,HG22,HG23 & CG2 & Decrease \\
\hline 1.22 & 18.83 & $\mathrm{~T} 18$ & HG21,HG22,HG23 & CG2 & Decrease \\
\hline 1.22 & 27.53 & K31 & HD2 & $\mathrm{CD}$ & Decrease \\
\hline 1.25 & 25.9 & K31 & HG2 & $\mathrm{CG}$ & Decrease \\
\hline
\end{tabular}




\begin{tabular}{|c|c|c|c|c|c|}
\hline 1.248 & 19.96 & V29 & HG21, HG22, HG23 & CG2 & Decrease \\
\hline 1.27 & 38.58 & E27 & HG3 & $\mathrm{CG}$ & Decrease \\
\hline 1.297 & 18.8 & T51 & HG21, HG22, HG23 & CG2 & Decrease \\
\hline 1.3 & 42.4 & L7 & HB3 & $\mathrm{CB}$ & Decrease \\
\hline 1.32 & 23.9 & T49 & HG21, HG22, HG23 & CG2 & Decrease \\
\hline 1.33 & 25.07 & T53 & HG21, HG22, HG23 & CG2 & Increase \\
\hline 1.34 & 19.37 & L12 & HG & CG & Increase \\
\hline 1.37 & 32.26 & L12 & HB2 & $\mathrm{CB}$ & Decrease \\
\hline 1.39 & 19.14 & $\mathrm{~T} 17$ & HG21, HG22, HG23 & CG2 & Increase \\
\hline 1.47 & 19.18 & $\mathrm{~T} 25$ & HG21, HG22, HG23 & CG2 & Decrease \\
\hline 1.49 & 15.65 & A24 & HB1, HB2, HB3 & $\mathrm{CB}$ & Decrease \\
\hline 1.5 & 19.5 & T55 & HG21, HG22, HG23 & CG2 & Decrease \\
\hline 1.51 & 22.47 & T44 & HG21, HG22, HG23 & CG2 & Increase \\
\hline 1.52 & 25.23 & I6 & HG11, HG12, HG13 & CG1 & Decrease \\
\hline 1.52 & 38.6 & E27 & HG2 & CG & Decrease \\
\hline 1.66 & 21.05 & A 20 & HB1, HB2, HB3 & $\mathrm{CB}$ & Decrease \\
\hline 1.68 & 26.27 & K50 & HD3 & $\mathrm{CD}$ & Decrease \\
\hline 1.7 & 23.07 & K4 & HG2 & $\mathrm{CG}$ & Decrease \\
\hline 1.71 & 15.94 & A 48 & HB1, HB2, HB3 & $\mathrm{CB}$ & Decrease \\
\hline 1.76 & 23.1 & K4 & HG3 & CG & Decrease \\
\hline 1.77 & 25.3 & L7 & $\mathrm{HG}$ & $\mathrm{CG}$ & Increase \\
\hline 1.78 & 26.83 & I6 & HG12 & CG1 & Increase \\
\hline 1.8 & 40.25 & K31 & HB2 & $\mathrm{CB}$ & Increase \\
\hline 1.83 & 30.16 & E27 & HB2 & $\mathrm{CB}$ & Decrease \\
\hline 1.87 & 26.7 & K4 & HD2 & $\mathrm{CD}$ & Constant \\
\hline 1.94 & 30.69 & K13 & HD2 & $\mathrm{CD}$ & Increase \\
\hline 1.95 & 37.22 & K13 & HB2 & $\mathrm{CB}$ & Decrease \\
\hline 1.96 & 34.22 & V54 & $\mathrm{HB}$ & $\mathrm{CB}$ & Decrease \\
\hline 1.97 & 26.67 & K50 & HD2 & $\mathrm{CD}$ & Decrease \\
\hline 2.01 & 26.63 & K28 & HB2 & $\mathrm{CB}$ & Decrease \\
\hline 2.04 & 30.86 & V30 & $\mathrm{HB}$ & $\mathrm{CB}$ & Increase \\
\hline 2.045 & 30.23 & K13 & HD3 & $\mathrm{CD}$ & Decrease \\
\hline 2.08 & 38.68 & K31 & HE3 & $\mathrm{CE}$ & Decrease \\
\hline 2.11 & 28.16 & Q2 & HB3 & $\mathrm{CB}$ & Increase \\
\hline 2.12 & 34.22 & K10 & HB2 & $\mathrm{CB}$ & Decrease \\
\hline 2.13 & 29.13 & E19 & HB2 & $\mathrm{CB}$ & Decrease \\
\hline 2.15 & 32.99 & K13 & HB3 & $\mathrm{CB}$ & Decrease \\
\hline 2.16 & 28.13 & Q2 & HB2 & CB & Decrease \\
\hline 2.19 & 27.73 & $\mathrm{E} 42$ & HB3 & $\mathrm{CB}$ & Decrease \\
\hline 2.24 & 22.47 & E56 & HB3 & CB & Increase \\
\hline 2.27 & 30.43 & M1 & HB2 & $\mathrm{CB}$ & Increase \\
\hline 2.27 & 31.13 & Q2 & HG3 & CG & Decrease \\
\hline 2.28 & 28.3 & V5 & $\mathrm{HB}$ & $\mathrm{CB}$ & Decrease \\
\hline 2.29 & 33.59 & K50 & HB3 & $\mathrm{CB}$ & Increase \\
\hline 2.3 & 14.21 & M1 & HE1, HE2, HE3 & $\mathrm{CE}$ & Decrease \\
\hline 2.308 & 25.77 & Q32 & HB3 & $\mathrm{CB}$ & Decrease \\
\hline 2.31 & 36.09 & I6 & $\mathrm{HB}$ & $\mathrm{CB}$ & Decrease \\
\hline
\end{tabular}




\begin{tabular}{|c|c|c|c|c|c|}
\hline 2.32 & 27.63 & E15 & HB2 & $\mathrm{CB}$ & Decrease \\
\hline 2.33 & 31.36 & E42 & $\mathrm{HG}$ & HG3 & Increase \\
\hline 2.37 & 25.9 & Q32 & HB2 & $\mathrm{CB}$ & Decrease \\
\hline 2.39 & 28.7 & V39 & HB2 & $\mathrm{CB}$ & Decrease \\
\hline 2.4 & 30.19 & M1 & HG3 & CG & Decrease \\
\hline 2.43 & 33.79 & M1 & HB3 & $\mathrm{CB}$ & Increase \\
\hline 2.47 & 31.59 & $\mathrm{E} 42$ & HG3 & CG & Increase \\
\hline 2.49 & 40.71 & D46 & HB3 & $\mathrm{CB}$ & Decrease \\
\hline 2.52 & 33.46 & E15 & HG3 & CG & Decrease \\
\hline 2.55 & 31.36 & $\mathrm{E} 42$ & HG2 & HG & Decrease \\
\hline 2.58 & 37.15 & E56 & HG2 & CG & Decrease \\
\hline 2.59 & 34.46 & Q32 & HG3 & $\mathrm{CG}$ & Decrease \\
\hline 2.66 & 31.33 & Q32 & HG2 & CG & Decrease \\
\hline 2.7 & 37.28 & N37 & HB2 & $\mathrm{CB}$ & Decrease \\
\hline 2.73 & 39.81 & N8 & HB3 & $\mathrm{CB}$ & Decrease \\
\hline 2.81 & 38.95 & Y45 & HB3 & $\mathrm{CB}$ & Increase \\
\hline 2.82 & 40.86 & D46 & HB2 & $\mathrm{CB}$ & Decrease \\
\hline 2.826 & 38.42 & D36 & HB2 & $\mathrm{CB}$ & Decrease \\
\hline 2.84 & 38.98 & Y3 & HB3 & $\mathrm{CB}$ & Increase \\
\hline 2.94 & 24.87 & T16 & HG1 & CG2 & Decrease \\
\hline 2.95 & 36.72 & N35 & HB2 & $\mathrm{CB}$ & Increase \\
\hline 2.96 & 37.75 & $\mathrm{D} 40$ & HB3 & $\mathrm{CB}$ & Decrease \\
\hline 3.005 & 38.82 & Y45 & HB2 & $\mathrm{CB}$ & Decrease \\
\hline 3.016 & 36.62 & N35 & HB3 & $\mathrm{CB}$ & Increase \\
\hline 3.06 & 37.82 & $\mathrm{D} 40$ & HB2 & $\mathrm{CB}$ & Decrease \\
\hline 3.08 & 39.58 & K4 & HE2 & $\mathrm{CE}$ & Decrease \\
\hline 3.135 & 39.55 & K13 & HE2 & $\mathrm{CE}$ & Increase \\
\hline 3.17 & 40.38 & K50 & HE2 & $\mathrm{CE}$ & Increase \\
\hline 3.19 & 37.38 & N8 & HB2 & $\mathrm{CB}$ & Decrease \\
\hline 3.2 & 56.69 & E27 & $\mathrm{HA}$ & $\mathrm{CA}$ & Increase \\
\hline 3.21 & 39.72 & K10 & HE2 & $\mathrm{CE}$ & Decrease \\
\hline 3.28 & 37.58 & F33 & HB3 & $\mathrm{CB}$ & Increase \\
\hline 3.28 & 52.43 & A23 & HA & $\mathrm{CA}$ & Decrease \\
\hline 3.29 & 39.75 & K13 & HE3 & $\mathrm{CE}$ & Decrease \\
\hline 3.3 & 40.48 & $\mathrm{D} 22$ & HB2 & $\mathrm{CB}$ & Decrease \\
\hline 3.32 & 37.48 & $\mathrm{~F} 33$ & HB2 & $\mathrm{CB}$ & Increase \\
\hline 3.37 & 39.78 & F52 & HB2 & $\mathrm{CB}$ & Decrease \\
\hline 3.41 & 40.75 & K50 & HE3 & $\mathrm{CE}$ & Decrease \\
\hline 3.45 & 28.26 & W43 & HB2 & $\mathrm{CB}$ & Decrease \\
\hline 3.47 & 39.65 & F52 & HB3 & $\mathrm{CB}$ & Decrease \\
\hline 3.51 & 64.37 & V30 & HA & CA & Decrease \\
\hline 3.62 & 34.62 & F34 & HB3 & $\mathrm{CB}$ & Decrease \\
\hline 3.64 & 39.68 & F34 & HB2 & $\mathrm{CB}$ & Decrease \\
\hline 3.74 & 63.02 & V29 & $\mathrm{HA}$ & $\mathrm{CA}$ & Decrease \\
\hline 3.77 & 53.03 & A26 & HA & CA & Decrease \\
\hline 3.82 & 63.05 & K31 & HA & $\mathrm{CA}$ & Decrease \\
\hline 3.87 & 57.29 & K28 & HA & CA & Decrease \\
\hline 3.88 & 63.68 & $\mathrm{~T} 25$ & HA & CA & Decrease \\
\hline 3.91 & 64.2 & $\mathrm{~T} 18$ & $\mathrm{HB}$ & $\mathrm{CB}$ & Decrease \\
\hline 3.96 & 72.64 & T51 & $\mathrm{HB}$ & $\mathrm{CB}$ & Increase \\
\hline 3.98 & 68.78 & T53 & $\mathrm{HB}$ & $\mathrm{CB}$ & Decrease \\
\hline 3.99 & 70.04 & T16 & $\mathrm{HB}$ & $\mathrm{CB}$ & Decrease \\
\hline 4 & 42.71 & G9 & HA3 & $\mathrm{CA}$ & Increase \\
\hline 4.018 & 58.86 & Q32 & $\mathrm{HA}$ & CA & Increase \\
\hline 4.118 & 56.56 & F34 & HA & $\mathrm{CA}$ & Decrease \\
\hline 4.12 & 44.21 & G38 & HA3 & $\mathrm{CA}$ & Decrease \\
\hline
\end{tabular}




\begin{tabular}{|c|c|c|c|c|c|}
\hline 4.14 & 62.35 & V39 & HA & $\mathrm{CA}$ & Decrease \\
\hline 4.19 & 55.96 & A24 & HA & $\mathrm{CA}$ & Decrease \\
\hline 4.21 & 65.45 & $\mathrm{~T} 25$ & $\mathrm{HB}$ & $\mathrm{CB}$ & Decrease \\
\hline 4.25 & 69.41 & $\mathrm{~T} 17$ & HA & CA & Decrease \\
\hline 4.26 & 42.71 & G41 & HA2 & $\mathrm{CA}$ & Decrease \\
\hline 4.3 & 61.65 & V21 & HA & $\mathrm{CA}$ & Decrease \\
\hline 4.31 & 44.31 & G38 & HA2 & $\mathrm{CA}$ & Decrease \\
\hline 4.32 & 69.54 & T55 & $\mathrm{HB}$ & $\mathrm{CB}$ & Decrease \\
\hline 4.34 & 55.63 & A48 & HA & $\mathrm{CA}$ & Increase \\
\hline 4.36 & 42.71 & G41 & HA3 & $\mathrm{CA}$ & Decrease \\
\hline 4.41 & 59.93 & K10 & HA & $\mathrm{CA}$ & Increase \\
\hline 4.42 & 58.82 & V54 & HA & $\mathrm{CA}$ & Decrease \\
\hline 4.43 & 54.13 & D47 & HA & CA & Increase \\
\hline 4.44 & 55.76 & M1 & HA & $\mathrm{CA}$ & Decrease \\
\hline 4.45 & 58.96 & E56 & HA & CA & Increase \\
\hline 4.49 & 59.22 & F33 & HA & CA & Increase \\
\hline 4.58 & 67.41 & $\mathrm{~T} 44$ & $\mathrm{HB}$ & $\mathrm{CB}$ & Decrease \\
\hline 4.64 & 59.12 & T49 & HA & $\mathrm{CA}$ & Increase \\
\hline 4.68 & 52.43 & D36 & HA & $\mathrm{CA}$ & Increase \\
\hline 4.74 & 52.03 & $\mathrm{D} 46$ & HA & $\mathrm{CA}$ & Increase \\
\hline 4.81 & 44.21 & G14 & HA & $\mathrm{CA}$ & Decrease \\
\hline 4.87 & 51.26 & E42 & HA & CA & Constant \\
\hline 4.99 & 42.41 & G9 & HA2 & $\mathrm{CA}$ & Decrease \\
\hline 5 & 50.4 & D22 & HA & CA & Decrease \\
\hline 5.11 & 53.5 & A20 & HA & CA & Decrease \\
\hline 5.16 & 54.73 & $\mathrm{Q} 2$ & HA & CA & Decrease \\
\hline 5.18 & 52.1 & L12 & HA & $\mathrm{CA}$ & Decrease \\
\hline 5.19 & 60.22 & $\mathrm{~T} 18$ & HA & $\mathrm{CA}$ & Decrease \\
\hline 5.21 & 57.92 & T55 & HA & $\mathrm{CA}$ & Decrease \\
\hline 5.29 & 51.8 & K13 & HA & $\mathrm{CA}$ & Decrease \\
\hline 5.31 & 55.26 & Y3 & HA & $\mathrm{CA}$ & Decrease \\
\hline 5.46 & 49.57 & N8 & HA & $\mathrm{CA}$ & Decrease \\
\hline 5.48 & 52.4 & E19 & HA & CA & Decrease \\
\hline 5.49 & 53.23 & W43 & HA & CA & Decrease \\
\hline 5.57 & 58.36 & T16 & HA & $\mathrm{CA}$ & Decrease \\
\hline 5.64 & 58.46 & $\mathrm{~T} 17$ & HA & CA & Decrease \\
\hline 5.66 & 53.26 & E15 & HA & $\mathrm{CA}$ & Decrease \\
\hline 5.76 & 59.42 & T51 & HA & $\mathrm{CA}$ & Decrease \\
\hline 6.07 & 54.46 & F52 & HA & $\mathrm{CA}$ & Decrease \\
\hline 6.27 & 118.5 & W43 & HZ3 & $\mathrm{CZ}$ & Decrease \\
\hline 6.52 & 115.4 & Y45 & HD1 & CD1 & Decrease \\
\hline 6.74 & 122.1 & W43 & $\mathrm{HH} 2$ & $\mathrm{CH} 2$ & Decrease \\
\hline 6.98 & 115.82 & $\mathrm{Y} 3$ & HE1 & $\mathrm{CE}$ & Increase \\
\hline 7.06 & 127.37 & F33 & $\mathrm{HZ}$ & $\mathrm{CZ}$ & Decrease \\
\hline 7.09 & 128.23 & F34 & HD1 & CD1 & Decrease \\
\hline 7.169 & 130.3 & F33 & HE1 & $\mathrm{CE}$ & Decrease \\
\hline 7.17 & 128.47 & F33 & HD1 & CD1 & Decrease \\
\hline 7.21 & 131.11 & Y3 & HD1 & CD1 & Constant \\
\hline 7.27 & 128.97 & F52 & HE1 & $\mathrm{CE}$ & Decrease \\
\hline 7.4 & 129.54 & W43 & HD1 & $\mathrm{CD}$ & Increase \\
\hline 7.51 & 130.2 & W43 & HG2 & CG & Increase \\
\hline 7.7 & 130.1 & F52 & HD1 & CD1 & Decrease \\
\hline
\end{tabular}


2D ${ }^{1} \mathrm{H}-{ }^{13} \mathrm{C}$ HSQC NMR spectra of GB1 HS\#124 ${ }^{\mathrm{F} 26 \mathrm{~A}}$ at different time points during the course of amyloid fibrillation. $2 \mathrm{D}{ }^{1} \mathrm{H}-{ }^{13} \mathrm{C}$ HSQC NMR spectra were recorded for GB1HS\#124F26A at time points $0 \mathrm{~h}, 36 \mathrm{~h}$ and $84 \mathrm{~h}$ at $58^{\circ} \mathrm{C}$ in sodium phosphate buffer (pH=5.5). The superimposed spectra for each time point are shown in figure $\mathrm{S} 2$ which shows changes in intensity of peaks.

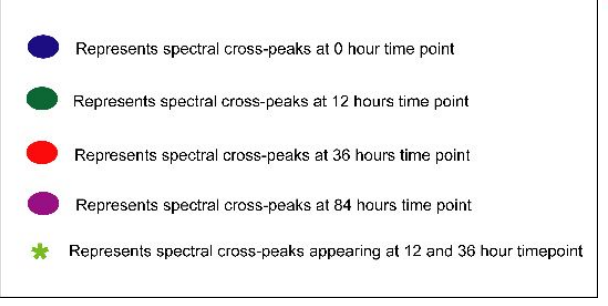

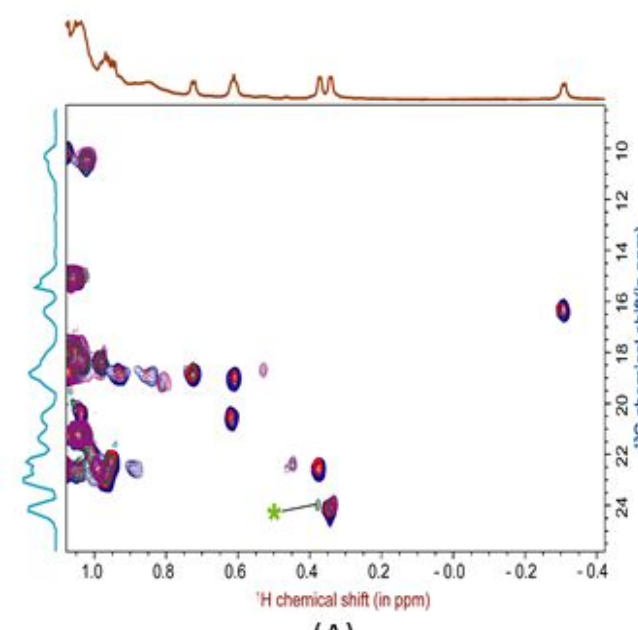

(A)

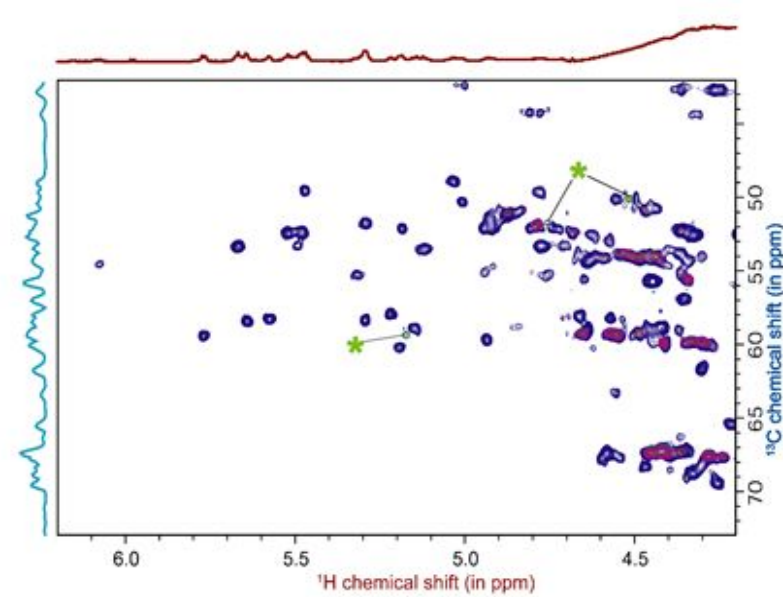

(D)

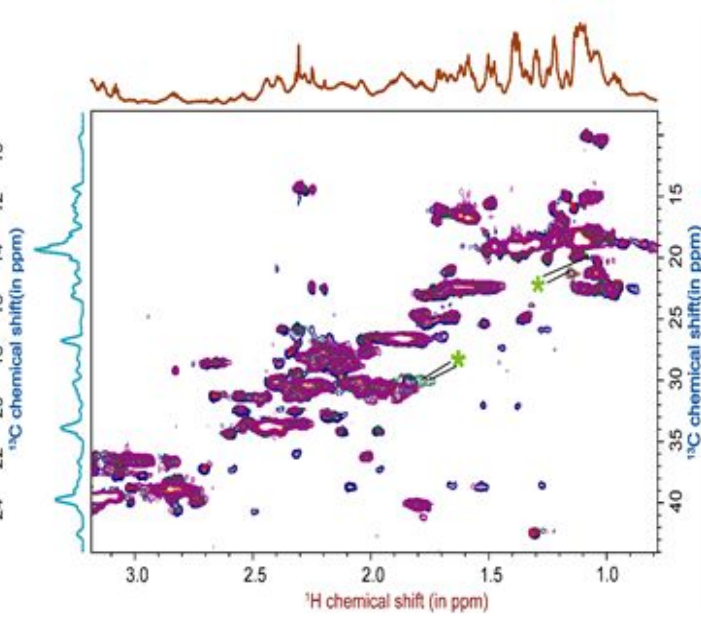

(B)

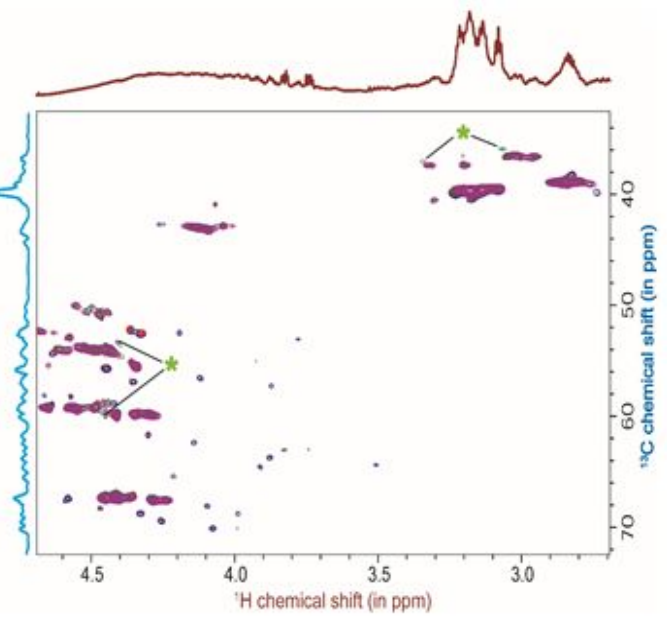

(C)

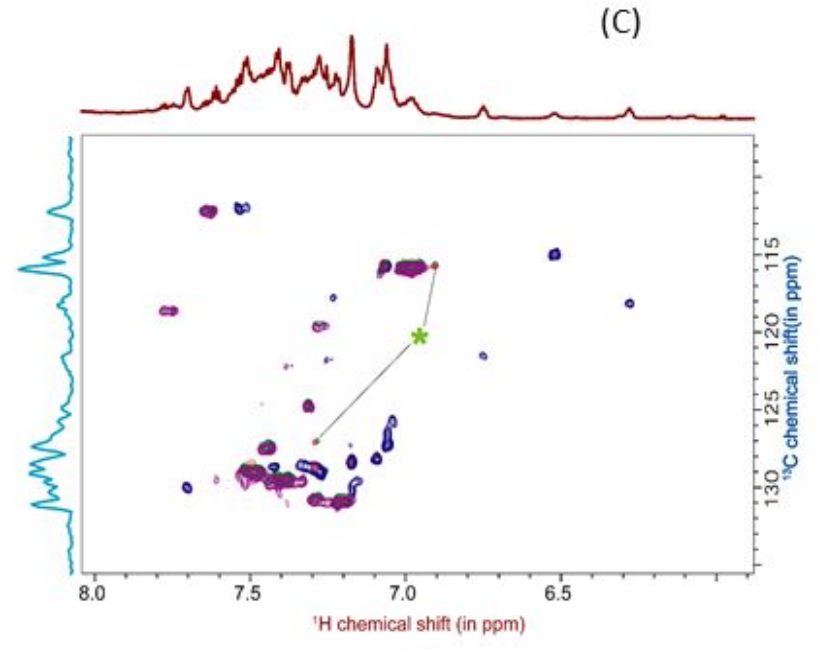

(E)

Figure S2: Superimposed 2D ${ }^{1} \mathrm{H}-{ }^{13} \mathrm{C}$ HSQC NMR spectra at $0 \mathrm{~h}, 36 \mathrm{~h}$ and $84 \mathrm{~h}$ timepoints of fibrillation of GB1HS\#124 ${ }^{\mathrm{F} 26 \mathrm{~A}}$ depicting (A) regions -0.4 to 1.0 ppm, (B) regions 1.0 to $3.0 \mathrm{ppm},(\mathrm{C})$ regions 3.0 to $4.5 \mathrm{ppm}$, (D) regions 4.5 to $6.0 \mathrm{ppm}$ and (E) regions 6.0 to $8.0 \mathrm{ppm}$ of ${ }^{1} \mathrm{H}$ chemical shift 


\section{Diffusion Coefficient for protons attached to side chain carbons of amino acid residues in GB1HS\#124 ${ }^{\mathrm{F} 2 \mathrm{~A}}$ peptide}

2D DOSY (ledbpgp2s) NMR spectra were obtained for ${ }^{1} \mathrm{H}$ nuclei at $58^{\circ} \mathrm{C}$. Diffusion coefficients for side chain protons were calculated by curve fitting using the equation

$$
\mathrm{I}=\mathrm{I}_{0} \mathrm{e}^{-\mathrm{D} \gamma 2 \mathrm{~g} 2 \delta 2(\Delta-\delta / 3)}
$$

Table S2: List of diffusion coefficient values for side chain protons

\begin{tabular}{|c|c|c|c|c|c|}
\hline & & \multicolumn{4}{|c|}{ Diffusion coefficient (in $\mathbf{m}^{2} \mathbf{s}^{-1}$ ) } \\
\hline $\begin{array}{l}\text { Amino acid residue-side-chain-attached } \\
\text { proton }\end{array}$ & $\begin{array}{l}{ }^{1} \mathbf{H} \\
\text { Chemical } \\
\text { shift (in } \\
\text { ppm) }\end{array}$ & at 0 hour & at 12 hours & at 36 hours & at 84 hours \\
\hline L12- HD21,22,23 & 0.37 & $2.594 \mathrm{E}-10$ & 2.36E-09 & $6.28 \mathrm{E}-10$ & $1.14 \mathrm{E}-11$ \\
\hline V30-HG21,22,23 & 0.616 & $6.87 \mathrm{E}-11$ & 2.71E-09 & $7.26 \mathrm{E}-11$ & $1.18 \mathrm{E}-11$ \\
\hline V54-HG11,12,13 & 0.72 & $3.73 \mathrm{E}-10$ & $5.54 \mathrm{E}-09$ & $7.13 \mathrm{E}-11$ & $1.16 \mathrm{E}-12$ \\
\hline L7-HD11,12,13 & 0.96 & $1.95 \mathrm{E}-10$ & 2.13E-09 & $2.1 \mathrm{E}-11$ & $1.93 \mathrm{E}-12$ \\
\hline T16 -HG11,12,13 & 1.116 & $2.23 \mathrm{E}-10$ & $2.73 \mathrm{E}-09$ & $2.16 \mathrm{E}-10$ & $2.2 \mathrm{E}-11$ \\
\hline I6-HG21,22,23 & 1.161 & $2.18 \mathrm{E}-10$ & $2.88 \mathrm{E}-09$ & $2.66 \mathrm{E}-11$ & $2.26 \mathrm{E}-12$ \\
\hline V29-HG21,22,23 & 1.248 & $2.09 \mathrm{E}-10$ & $2.6 \mathrm{E}-09$ & $2.63 \mathrm{E}-11$ & $2.547 \mathrm{E}-12$ \\
\hline E27-HG3 & 1.277 & $2.07 \mathrm{E}-10$ & $2.42 \mathrm{E}-09$ & $2.04 \mathrm{E}-11$ & $2.07 \mathrm{E}-12$ \\
\hline T49 -HG21,22,23 & 1.322 & $2.08 \mathrm{E}-10$ & $2.51 \mathrm{E}-09$ & $1.79 \mathrm{E}-11$ & $1.68 \mathrm{E}-12$ \\
\hline L12-HB2 & 1.37 & $2.16 \mathrm{E}-10$ & $2.8 \mathrm{E}-09$ & $2.08 \mathrm{E}-11$ & $1.97 \mathrm{E}-12$ \\
\hline $\begin{array}{c}\text { T55/T44-HG21, HG22, HG23/HG21, } \\
\text { HG22, HG23 }\end{array}$ & 1.52 & $1.97 \mathrm{E}-10$ & 1.33E-09 & $2.16 \mathrm{E}-11$ & $2.17 \mathrm{E}-12$ \\
\hline K50-HD3 & 1.683 & $2.06 \mathrm{E}-11$ & $2.67 \mathrm{E}-10$ & $2.27 \mathrm{E}-11$ & $2.05 \mathrm{E}-12$ \\
\hline A48-HB1, HB2, HB3 & 1.713 & $2.11 \mathrm{E}-10$ & $2.52 \mathrm{E}-09$ & $2.179 \mathrm{E}-11$ & $2.143 \mathrm{E}-12$ \\
\hline K4 -HG3 & 1.76 & $1.99 \mathrm{E}-10$ & $2.43 \mathrm{E}-09$ & $2.14 \mathrm{E}-11$ & $2.09 \mathrm{E}-12$ \\
\hline K13-HD3 & 2.045 & $2.04 \mathrm{E}-10$ & $2.68 \mathrm{E}-09$ & $2.23 \mathrm{E}-11$ & $2.04 \mathrm{E}-12$ \\
\hline K13-HB3 & 2.141 & $2.04 \mathrm{E}-10$ & 2.59E-09 & $2.2 \mathrm{E}-11$ & $2.12 \mathrm{E}-12$ \\
\hline E42-HB3 & 2.19 & $2.11 \mathrm{E}-10$ & 1.64E-09 & 2.19E-11 & $1.95 \mathrm{E}-12$ \\
\hline M1-HE1, HE2, HE3 & 2.301 & $2.17 \mathrm{E}-10$ & 2.75E-09 & $2.05 \mathrm{E}-11$ & $1.972 \mathrm{E}-12$ \\
\hline E42-HG2 & 2.331 & $2.08 \mathrm{E}-10$ & 2.63E-09 & $2.06 \mathrm{E}-11$ & $2.01 \mathrm{E}-12$ \\
\hline M1-HG3 & 2.411 & $2.13 \mathrm{E}-10$ & 2.67E-09 & $2.16 \mathrm{E}-11$ & $2 \mathrm{E}-12$ \\
\hline E42-HG2 & 2.57 & $1.46 \mathrm{E}-10$ & 1.17E-09 & $1.1 \mathrm{E}-10$ & $3.3 \mathrm{E}-11$ \\
\hline K4-HE2 & 3.08 & $1.92 \mathrm{E}-10$ & 2.4E-09 & $2.14 \mathrm{E}-11$ & $1.55 \mathrm{E}-12$ \\
\hline D27-HA & 3.207 & $2.19 \mathrm{E}-10$ & $1.92 \mathrm{E}-09$ & $2.26 \mathrm{E}-11$ & $2.06 \mathrm{E}-12$ \\
\hline K10- HE2 & 3.255 & $1.92 \mathrm{E}-10$ & 2.37E-09 & $2.676 \mathrm{E}-11$ & $2.076 \mathrm{E}-12$ \\
\hline T18-HB & 3.942 & $1.58 \mathrm{E}-10$ & $1.29 \mathrm{E}-09$ & $2.17 \mathrm{E}-10$ & $3.28 \mathrm{E}-11$ \\
\hline T53-HB & 3.976 & $3.653 \mathrm{E}-10$ & 4.99E-09 & $1.25 \mathrm{E}-10$ & $7.62 \mathrm{E}-12$ \\
\hline F34-HA & 4.118 & $2.01 \mathrm{E}-10$ & 3.39E-09 & $3.19 \mathrm{E}-11$ & $2.29 \mathrm{E}-12$ \\
\hline V39-HA & 4.14 & $2.01 \mathrm{E}-10$ & $3.07 \mathrm{E}-09$ & $3.33 \mathrm{E}-11$ & $2.29 \mathrm{E}-12$ \\
\hline T25-HB & 4.21 & $2.09 \mathrm{E}-10$ & 3.39E-09 & $4.53 \mathrm{E}-11$ & $3.12 \mathrm{E}-12$ \\
\hline G41-HA2 & 4.26 & $2.16 \mathrm{E}-10$ & $3.27 \mathrm{E}-09$ & $3.4 \mathrm{E}-11$ & $2.42 \mathrm{E}-11$ \\
\hline G38-HA2 & 4.31 & 2.29E-10 & $3.26 \mathrm{E}-09$ & $2.63 \mathrm{E}-11$ & $1.823 \mathrm{E}-12$ \\
\hline V54-HA & 4.42 & $2.33 \mathrm{E}-10$ & 2.82E-09 & $2.557 \mathrm{E}-11$ & $2.151 \mathrm{E}-12$ \\
\hline G9-HA2 & 4.99 & 7.29E-10 & $6.28 \mathrm{E}-09$ & $1.09 \mathrm{E}-11$ & $1.09 \mathrm{E}-12$ \\
\hline D22-HA & 5.11 & $8.73 \mathrm{E}-10$ & $1.26 \mathrm{E}-09$ & $1.82 \mathrm{E}-10$ & $1.84 \mathrm{E}-11$ \\
\hline
\end{tabular}




\begin{tabular}{|c|c|c|c|c|c|}
\hline Q2-HA & 5.16 & $4.28 \mathrm{E}-10$ & $6.8 \mathrm{E}-09$ & $2.34 \mathrm{E}-10$ & $2.29 \mathrm{E}-11$ \\
\hline T55-HA & 5.21 & $3.46 \mathrm{E}-10$ & $5.89 \mathrm{E}-09$ & $3.33 \mathrm{E}-11$ & $3.6 \mathrm{E}-12$ \\
\hline F52-HA & 6.07 & $2.22 \mathrm{E}-10$ & $2.88 \mathrm{E}-09$ & $6.21 \mathrm{E}-11$ & $2.83 \mathrm{E}-11$ \\
\hline Y45-HE1 & 6.27 & $2.48 \mathrm{E}-10$ & $3.7 \mathrm{E}-09$ & $1.16 \mathrm{E}-11$ & $9.2 \mathrm{E}-12$ \\
\hline F33-HZ & 7.06 & $2.08 \mathrm{E}-10$ & $2.4 \mathrm{E}-09$ & $1.46 \mathrm{E}-11$ & $1.68 \mathrm{E}-12$ \\
\hline F34-HD1 & 7.09 & $1.98 \mathrm{E}-10$ & $2.32 \mathrm{E}-09$ & $1.47 \mathrm{E}-11$ & $1.4 \mathrm{E}-12$ \\
\hline F33-HE1, HD1 & 7.17 & $2.11 \mathrm{E}-10$ & $2.37 \mathrm{E}-09$ & $1.3 \mathrm{E}-11$ & $1.24 \mathrm{E}-12$ \\
\hline F52-HE1 & 7.27 & $2.13 \mathrm{E}-10$ & $2.66 \mathrm{E}-09$ & $1.69 \mathrm{E}-11$ & $1.83 \mathrm{E}-12$ \\
\hline
\end{tabular}




\section{T1 Relaxation measurements of side-chain-attached protons during fibrillation.}

In figure S3 T1 relaxation time values of side chain protons $\mathrm{HB}, \mathrm{HG}$ and $\mathrm{HD}$ in different structural elements of the peptide ( $\beta 1$ strand, $\beta 2$ strand, $\alpha$ - helix, $\beta 3$ strand and $\beta 4$ strand) have been compared. The values of $T 1$ for the dimeric chemical shifts of protons which do not experience any overlap of chemical shifts with that of the other soluble species were taken into consideration for analysis. Each of these dimeric chemical shifts assigned to the side-chain-attached protons experienced a different trend of $T 1$ relaxation time changes which explains behaviour of each of these side chain entities during the process of fibrillation through domain swapping. $T 1$ relaxation time values have been plotted as a bar graph as shown in figure S3, where a comparison of these values at time points 0 hour, 12 hours, 36 hours, and 84 hours could reveal certain features of the spin-lattice relaxation phenomenon of the side-chain-attached protons of amino acids in the structural elements constituting the peptide.

$T 1$ values falling under the range of 0.1-5 s were taken into consideration for the comparison. $\mathrm{T} 1$ values larger than $5 \mathrm{~s}$ were not taken into consideration as those may represent motion due to factors other than those responsible for spin-lattice relaxation. For most of the side-chain-attached protons in the peptide, the values of $T 1$ do not exhibit any increase or decrease in values of $T 1$. Values of $T 1$ relaxation time also do not remain constant on observation through the four time-points. 

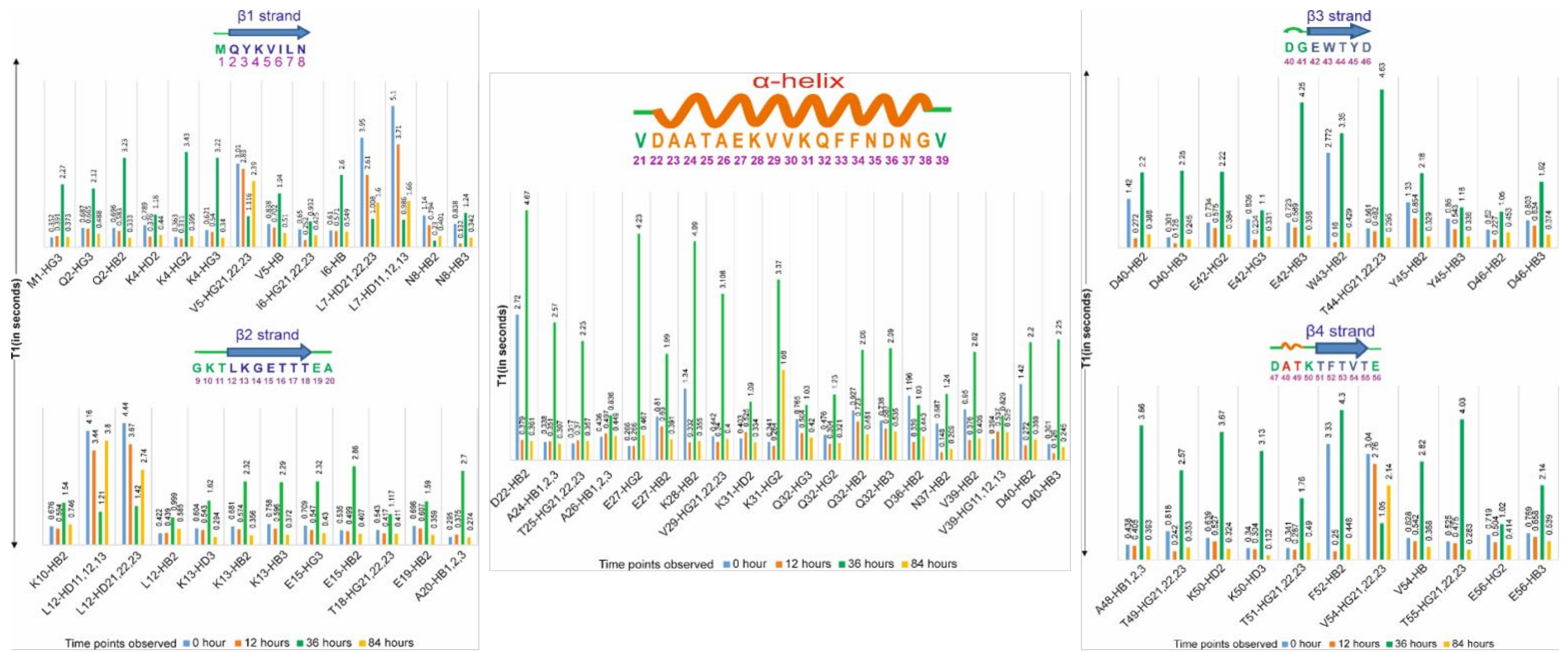

Time points observed $=0$ hour $\approx 12$ hours $\approx 36$ hours $=84$ hours

Figure S3 (A) T1 values (in seconds) plotted as a bar graph for side-chain-attached protons in the peptide segment 1-8 and 9-20, (B) the peptide segment 21-39 and (C) the peptide segment 40-46 and 47-56 at four time points of fibrillation i.e., 0 hour, 12 hours, 36 hours, and 84 hours. 
Most of the HB, HG and HD protons exhibit a sharp increase in values of $T 1$ at the 36- hour time point of fibrillation. In $\beta 1$ strand spanning 2 to 8 amino acids lead by turn region amino acid M1 of the GB1 HS\#124 ${ }^{\mathrm{F} 26 \mathrm{~A}}$ peptide (see figure S3(A)), HG protons of V5, HD protons of L7 and HB2 protons of N8 exhibit minimum $T 1$ values at 36-hour time point, while the other protons observed in this region at the 36-hour time point show maximum $T 1$ relaxation time values for each of the protons. In peptide region 9-20 containing $\beta 2$ strand flanked by turn regions of the GB1HS\#124 ${ }^{\mathrm{F} 26 \mathrm{~A}}$ peptide (see Figure S3(A)), HD protons of L12 show minimum $T 1$ values at 36-hour time point. For rest of the observed protons in this region, $T 1$ values peak at 36-hour time point. In the peptide region 21-39 which is folded into an $\alpha$-helix (see figure S3(B)), the T1 values peak at 36-hour time point for all the observed protons. In peptide region 40-46 constituting the $\beta 3$ strand flanked by $\beta$-turns (see figure S3(C)), all the observed protons show peaking values of $T 1$ relaxation time at 36-hour time point. The peptide region 47-56 constituting a $\beta$-turn and the $\beta 4$ strand (see figure $\mathrm{S} 3(\mathrm{C})$ ) shows a dip in $T 1$ values at 36-hour time point for HG protons of V54, except which all the other values observed for protons in these regions show maximum $T 1$ relaxation time at 36-hour time point. There may also exist contributions from bulk water and spin diffusion along with the slow chemical exchange could result in such high values of $\mathrm{T} 1$. 